\title{
Why there are many cycles of opening and closing of ground during an earthquake?
}

Yuhan Lin, Jess Lan Ouyang, Min Fu, Jingli Peng, Man Tang, Qiuyun Liu*

School of Life Sciences, Sun Yat-Sen University, Guangzhou 510275, China.

*Correspondence author: Qiuyun Liu, School of Life Sciences, Sun Yat-sen University, Guangzhou 510275, China.

E-mail address: liuqiuyunsysu@ 163.com (Q. Liu) 


\begin{abstract}
Both vertical and horizontal components of the strain are common for earthquakes, manifested in the forms of horizontal and upward movements of the earth crust. Horizontal component generates gaps in Point A, and gaps are subsequently closed by horizontal component in Point B. Reiteration of the cycles gives rise to the opening/closing phenomena seen on the ground hit by earthquakes.
\end{abstract}

Keywords: vertical and horizontal components; gaps; opening; closing 
During the 2008 Wenchuan earthquake in Sichuan, China, people witnessed the opening and closing of the ground for several consecutive times. To simplify the situation, we just consider solar gravitational pull.

At 6:00 am local time, solar pull generates tangential force only, namely, the horizontal component of the strain, with incrementally increasing strain straight down the Earth crust giving increased gravity. At 12:00 noon, solar pull produces vertical component of the strain only. Solar pull at 9:00 am may be the average of the above, and yields both vertical and horizontal components of the strain which are common for earthquakes (Fig. 1). With seismic activity starts at focal depth - Point A, the horizontal component generates gaps on the earth surface. The horizontal component at Point $\mathrm{B}$ then closes the gaps. Point $\mathrm{C}$ opens the gaps, and Point $\mathrm{D}$ closes the gaps. Reiteration of the process generates the opening/closing phenomena seen amid earthquakes.

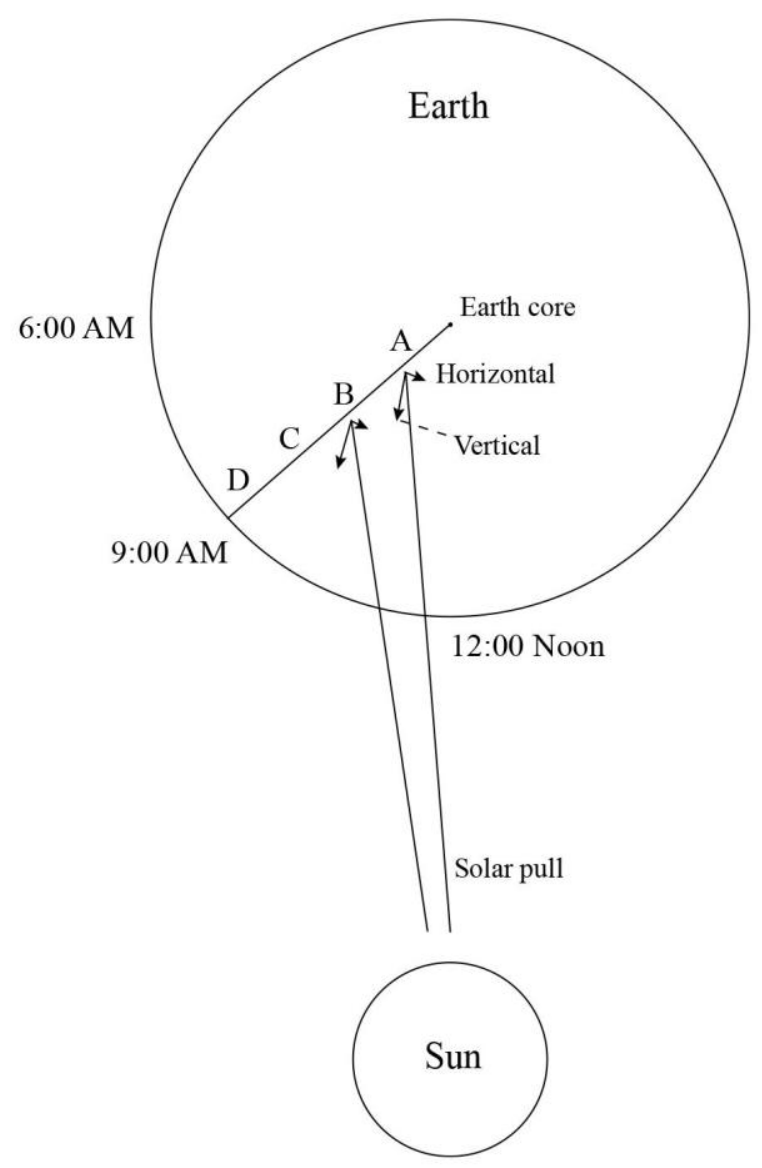

Fig. 1 Schematic view of opening/closing phenomena seen amidst earthquakes

\section{Conflict of interest}

None declared.

\section{ACKNOWLEDGMENT}


This work was supported by the Science and Technology Transformation Program of Sun Yat-sen University of China (33000-18843234) and Guangzhou Science and Technology Program (201804010328) to Q. Liu. We thank Yan Shi for editing.

\section{References}

1. Yan $\mathrm{S}, \mathrm{Du}, \mathrm{Y}, \mathrm{Xu} \mathrm{H}$, et al. Filtering out non-specific animal behavior to better predict impending earthquakes. OSF Preprints. 2020. https://doi.org/10.31219/osf.io/bs3qd.

2. Yan S, Wang X, Qi J, Liu Q. Volcanoes or earthquakes: Wrist wrestling. Science, 2019; http://science. sciencemag.org/content/358/6370/1520/tab-e-letters.

3. Yan S, Li J, Liu Q. Earthquakes-an intricate trio dance of gravitation. Science 2018; http://science. sciencemag.org/content/354/6315/1027/tab-e-letters.

4. Zhong H, Wang K, Shi Y, et al. Tsunamis is a prime example of the vertical component of the forces during earthquakes. Science 2017; http://science.sciencemag.org/content/358/6367/1164/tab-eletters. 